\title{
Polysomnographic evaluation of obstructive sleep apnea treatment with fixed pressure CPAP determined by formula
}

\author{
Danilo M. Sá1 ${ }^{10} \cdot$ Elcio O. Vianna ${ }^{1}[0$
}

Received: 25 July 2021 / Revised: 2 February 2022 / Accepted: 9 February 2022 / Published online: 4 March 2022

(c) The Author(s), under exclusive licence to Springer Nature Switzerland AG 2022

\begin{abstract}
Purpose The use of continuous positive airway pressure (CPAP) is one of the therapeutic modalities for obstructive sleep apnea (OSA). Manual titration polysomnography and the 90th or 95th percentiles of pressure titrated by automatic CPAP (APAP) are the current standard for determining fixed pressure. Pressures programmed at an arbitrary fixed value, or at preset values based on body mass index (BMI) or by predictive formulas, are presented as alternative forms. This study aimed to evaluate the residual apnea-hypopnea index (r-AHI) in polysomnography with CPAP therapy using pressure determined by formula and assess its feasibility to start treatment.

Methods Patients referred for CPAP therapy were followed up in three outpatient assessments and underwent polysomnography study with pressure CPAP obtained by formula.

Results The study sample consisted of 80 patients, 41 women; age $58.6 \pm 11.3$ years, BMI $34.1 \pm 7.5 \mathrm{~kg} / \mathrm{m}^{2}$ and cervical circumference $42.0 \pm 4.2 \mathrm{~cm}$. Most patients $(74 \%)$ had severe OSA and Epworth sleepiness scale (ESS) of $12.0 \pm 5.7$ points. The calculated average pressure was $7.8 \pm 2.1 \mathrm{cmH}_{2} \mathrm{O}$. Polysomnography studies showed an $\mathrm{r}-\mathrm{AHI}$ of $6.1 \pm 5.2$ events/h and reduction of $84 \%$ from baseline AHI. The r-AHI in the REM-supine was $8.4 \pm 9.9$ events/h. At 30- and 120-day follow-up assessment, adherence to CPAP was $78 \%$ and $75 \%$ and the ESS score was 6.9 and 6.1 points, respectively.

Conclusion Results suggest that a formula provides an effective initial pressure in the majority of patients (73\%). This simplified approach appears to be a viable alternative, with reductions in waiting lists and time from diagnosis to initiation of therapy.
\end{abstract}

Keywords Obstructive sleep apnea $\cdot$ CPAP $\cdot$ Residual apnea-hypopnea index $\cdot$ Predictive formula

$\begin{array}{ll}\text { Abbreviations } \\ \text { AASM } & \text { American academy of sleep medicine } \\ \text { AHI } & \text { Apnea and hipopnea index } \\ \text { APAP } & \text { Automatic positive airway pressure } \\ \text { BMI } & \text { Body mass index } \\ \text { B-PSG } & \text { Baseline polysomnography } \\ \text { CC } & \text { Cervical circumference } \\ \text { CPAP } & \text { Continuous positive airway pressure } \\ \text { EDS } & \text { Excessive daytime sleepniness } \\ \text { ESS } & \text { Epworth sleepiness scale }\end{array}$

Research carried out at Hospital das Clínicas, Medical School of Ribeirão Preto, University of São Paulo, Ribeirão Preto (SP) Brazil.

Danilo M. Sá

danilosa@usp.br

1 Department of Medicine, Medical School of Ribeirão Preto, University of São Paulo, Av. Bandeirantes, 3900 - Ribeirão Preto, São Paulo 14048-900, Brazil
NREM Non rapid eyes movement

OSA Obstructive sleep apnea

Peff Effective pressure

PLM Periodic limb movement

PSG Polysomnography

r-AHI Residual apnea and hipopnea index

REM Rapid eyes movement

\section{Introduction}

Obstructive sleep apnea (OSA) is characterized by episodes of upper airway obstruction during sleep, causing arousals, and excessive daytime sleepiness (EDS) [1], with different therapeutic modalities available [2]. Continuous positive airway pressure (CPAP) therapy uses a fixed pressure defined by manual titration polysomnography (gold standard method), automatic CPAP (APAP) titration, arbitrarily at a 
fixed value or by calculating pressure from predictive formulas [3-7].

Initially, the pressure of predictive formulas was considered as an initial value in manual titration to have less incremental changes $[8,9]$. Subsequently, the use of formulas was extrapolated to define the initial treatment pressure without titration polysomnography [10]. In 2015, a review presented cataloged formulas [7].

Although extrapolation to clinical practice has been questioned [11], the pressure predicted by the Miljeteig and Hoffstein formula was equivalent to manually titrated or autotitrated pressure in terms of outcomes such as the Epworth sleepiness scale (ESS), residual AHI, arousal index, percentage of sleep periods, and oxygen saturation [12].

This research aimed to evaluate the polysomnographic and clinical findings of CPAP therapy programmed with fixed pressure obtained by this formula. Our hypothesis was that the formula-derived CPAP setting would produce results of sufficient quality to justify use as the initial treatment of OSA.

\section{Methods}

This was an intervention study involving 80 patients to determine the success rate of CPAP therapy using pressure predicted by the Miljeteig and Hoffstein formula. The calculation of sample size was based on the success prevalence of manual CPAP titration, estimated at approximately $70 \%$ [13-15], an absolute tolerable error of $10 \%$, and $95 \%$ confidence coefficient. Volunteer patients gave signed informed consent which was approved by the institution's research ethics committee.

Outpatients diagnosed with OSA on baseline polysomnography with indication for CPAP therapy and without previous use of any device were sequentially included. Mild OSA was defined as an AHI between 5 and 14.9, moderate as an $\mathrm{AHI}$ between 15 and 29.9, and severe as an AHI equal to or greater than 30 [16].

In the first outpatient assessment, anthropometric and clinical data were collected. CPAP devices had the pressure programmed at a fixed value according to the result of the formula $[(0.13 * \mathrm{CC})+(0.04 * \mathrm{AHI})+(0.16 * \mathrm{BMI})-5.12$ ], composed of the values of cervical circumference (CC), apnea and hypopnea index (AHI), and body mass index (BMI) $[8,9]$.

In outpatient follow-up assessments at 30 and 120 days, the usage data provided by the CPAP device were collected. In the second month of treatment, they underwent a full night polysomnography using CPAP with the initial pressure schedule.

Pressure was not reviewed or changed during follow-up to the end of the research protocol in the 120-day follow-up assessment. The authors did not perform a manual titration to achieve effective pressure for treatment in these patients as part of the research protocol. All patients treated at the institution were referred to await manual titration on the waiting list.

The evaluation of the quality of pressure estimated by formula for the control of obstructive respiratory events in polysomnography with CPAP was performed according to the grading system proposed by Hirshkowitz and Sharafkhaneh with the following terms: optimal, good, adequate, and inadequate pressure, whose main parameter was the residual AHI value during the supine REM stage.

\section{Statistical analysis}

Baseline and treatment polysomnography data were compared using the paired Student's $t$ test for quantitative variables or McNemar's test for qualitative variables. Poisson regression models with robust variance were used to assess the association of acceptable CPAP pressure with demographic and clinical variables, because it provides a better estimate of the prevalence ratio more significantly for crosssectional studies [17]. Linear models of mixed effects with variance matrix and unstructured covariance, adjusted by baseline measurements were used to evaluate the longitudinal changes of the ESS score.

\section{Results}

Demographic and anthropometric data, comorbidities, sleep characteristics, and baseline polysomnography are shown in Table 1. All 80 patients diagnosed with OSA underwent polysomnography with CPAP at fixed pressure calculated throughout the night and had the residual AHI determined (Fig. 1).

Most patients (79\%) used a nasal mask and the mean pressure calculated by the formula was $7.8 \pm 2.1 \mathrm{cmH}_{2} \mathrm{O}$. Table 2 presents the comparative data between baseline and treatment polysomnography. Polysomnographs with CPAP showed a general residual AHI (r-AHI) of $6.1 \pm 5.2$ events/h, a reduction in the baseline AHI value by $84 \% \pm 17.3$ events/h; $50 \%$ of the patients had $\mathrm{r}-\mathrm{AHI}<5$ events/h and $82 \%$ ( 65 patients) had $\mathrm{r}-\mathrm{AHI}<10$ events/h. The r-IAH in the supine position during the REM stage was $8.4 \pm 9.9$ events/h. The $\mathrm{r}-\mathrm{AHI}$ of patients using a nasal mask (nCPAP) and oronasal mask (oCPAP) were 5.6 \pm 5.1 and $8.0 \pm 5.3$, respectively.

Only four exams were within the definition of inadequate pressure. Of the other exams, 18 had an adequate grade, and 30 and 28 met the criteria of good and optimal, respectively. 
Table 1 Characteristics of the volunteers $(n=80)$

\begin{tabular}{|c|c|}
\hline Variable & $N(\%)$ or mean $(\mathrm{sd})$ \\
\hline Sex (male) & $39(49 \%)$ \\
\hline Age (year) & $58.6 \pm 11.3$ \\
\hline Weight (kg) & $91.6 \pm 22.7$ \\
\hline BMI $\left(\mathrm{kg} / \mathrm{m}^{2}\right)$ & $34.1 \pm 7.5$ \\
\hline Smoking & $6(8 \%)$ \\
\hline Ex-smoking & $19(24 \%)$ \\
\hline No smoking & $55(69 \%)$ \\
\hline COPD & $7(9 \%)$ \\
\hline Asthma & $11(14 \%)$ \\
\hline Systemic hypertension & $50(63 \%)$ \\
\hline Epilepsy & $3(4 \%)$ \\
\hline Restless legs syndrome & $5(6 \%)$ \\
\hline Benzodiazepine & $12(15 \%)$ \\
\hline SSRI & $34(43 \%)$ \\
\hline Zolpidem & $3(4 \%)$ \\
\hline Tricyclic antidepressant & $9(11 \%)$ \\
\hline Anticonvulsant & $6(8 \%)$ \\
\hline Pregabalin/gababentin & $6(75 \%)$ \\
\hline \multicolumn{2}{|l|}{ Tonsils } \\
\hline Grade I & $62(78 \%)$ \\
\hline Grade II & $14(18 \%)$ \\
\hline Grade III & $3(4 \%)$ \\
\hline Grade IV & $1(1 \%)$ \\
\hline \multicolumn{2}{|l|}{ m-Mallampatti } \\
\hline Grade I & $9(11 \%)$ \\
\hline Grade II & $14(18 \%)$ \\
\hline Grade III & $15(19 \%)$ \\
\hline Grade IV & $29(36 \%)$ \\
\hline Cervical circumference $(\mathrm{cm})$ & $42.0 \pm 4.2$ \\
\hline Female circumference $(\mathrm{cm})$ & $39.8 \pm 3.6$ \\
\hline Male circumference $(\mathrm{cm})$ & $44.4 \pm 3.3$ \\
\hline Mean Saturation & $97 \% \pm 2$ \\
\hline \multicolumn{2}{|l|}{ Severity - OSA (events/h) } \\
\hline Severe OSA & $59(74 \%)$ \\
\hline Moderate OSA & $19(24 \%)$ \\
\hline Mild OSA & $2(3 \%)$ \\
\hline \multicolumn{2}{|l|}{ AHI (events/h) } \\
\hline Baseline AHI & $49.5 \pm 9.9$ \\
\hline Baseline REM AHI & $45.9 \pm 36.0$ \\
\hline Baseline supine AHI & $55.1 \pm 43.5$ \\
\hline
\end{tabular}

$B M I$, body mass index; $C P O D$. chronic obstructive pulmonary disease; SSRI, selective serotonin reuptake inhibitors; OSA, obstructive sleep apnea; $A H I$, apnea and hypopnea index; REM, rapid eye moviment

Therefore, 58 polysomnographies using CPAP had pressure with an acceptable grade (sum of good and optimal), which represents $73 \%$ of the total sample.

The comparisons between the group with acceptable and with unacceptable CPAP pressure showed that only the

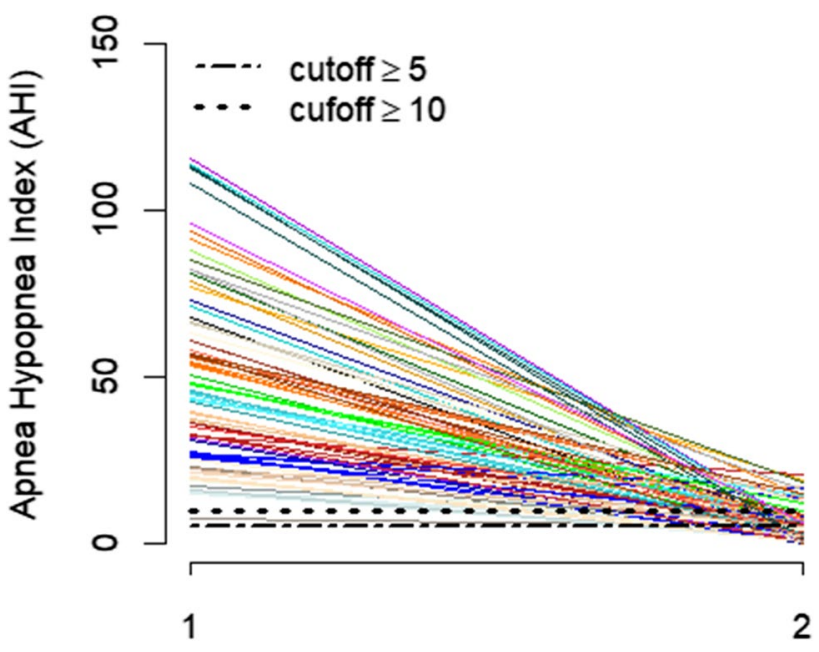

Baseline AHI (1) vs Residual AHI (2)

Fig. 1 Individual responses of residual AHI and cut-off values of 5 events/h and 10 events/h. AHI, apnea and hypopnea index

variable weight (>105 kg), type of mask (nasal), presence of diabetes, and 30-day assessment ESS below 10 points had a significant association with acceptable pressure quality (Table 3).

Analysis was performed to separate patients without residual respiratory events $(\mathrm{r}-\mathrm{AHI}<5 / \mathrm{h})$ from those with residual respiratory disorders (cutoff of $=/>5 / \mathrm{h}$ and a cutoff of $=/>10 / \mathrm{h}$ ) (Table 4). Comparison between patients with residual AHI less than or equal to 10 events/h and AHI greater than 10 events/h is shown in (Table 5).

Patients on treatment whose pressure met the acceptable criterion had a 3.61 times chance of not having excessive daytime sleepiness than those whose pressure was considered unacceptable (Table 6). This association did not present significant findings for patients whose pressure was able to decrease the residual AHI below five or below $15 / \mathrm{h}$. The ESS in each assessment of the follow-up was 6.9 and 6.1 points. The Epworth values in the 1st assessment and in the 2nd assessment showed a significant reduction of 5.1 and 5.9 points in relation to the baseline ESS (Table 7).

Adherence to CPAP ( $>4.0 \mathrm{~h} / \mathrm{night})$ was $78 \%$ in the in the 30-day follow-up assessment and 75\% in the 120-day followup assessment. Only $14 \%$ of patients did not show adherence in any of the follow-up assessments.

\section{Discussion}

The residual AHI of our study (6.1 events/h) is in the range of five to 10 events $/ \mathrm{h}$, with $50 \%$ of the patients presenting r-AHI below 5 events/h and 65 patients (82\%) presenting r-AHI below 10 events/h, in agreement with studies that 
use the term effective treatment pressure based on residual events up to the value of 10 events/h $[18,19]$. The same understanding applies to the other residual AHI subtypes, such as $\mathrm{r}-\mathrm{AHI}$ in the supine position $(7.2 / \mathrm{h}), \mathrm{r}-\mathrm{AHI}$ in REM sleep $(9.5 / \mathrm{h})$, and $\mathrm{r}-\mathrm{AHI}$ in supine REM $(8.4 / \mathrm{h})$.

Lloberes et al. showed similar results, with r-AHI of 5.6/h in the evaluation of CPAP effect on the control of residual obstructive events and on sleep architecture [20]. Our findings must be considered as having clinical impact and therapeutic success, especially when oximetry, sleep architecture, and sleepiness scale show excellent response and reach values of normality, associated with high degree of adherence to CPAP treatment.

In addition, our data showed that the r-AHI of patients using a nasal mask was lower compared to those using an oronasal mask. Therefore, the use of this formula may be less effective in determining pressure in patients who are candidates for the use of an oronasal mask compared to a nasal mask.

The effect of CPAP treatment on sleep architecture, sleep efficiency, and arousal index showed significant improvement. There was no change in the N3 and REM stages. Previous use of CPAP for at least 30 days prior to research polysomnography may reduce or eliminate the rebound effect of slow-wave N3 sleep and REM sleep, which is more often seen as a first-night effect in acute treatment with CPAP [21].

The parameters for analysis of residual OSA (r-OSA) and the cut-off values for residual AHI are still uncertain. According to manual titration guidelines, effective pressure is that capable of controlling obstructive events to preferentially obtain r-AHI below 5 events/h, associated with saturation above $90 \%$. Another definition of residual OSA is the presence of r-AHI above 5 events/h on polysomnography with auto-CPAP [22]. Desai et al. evaluated the accuracy of the APAP device in estimating the residual AHI using two cut-off values (5 and 10). The cut-off point of 10 events/h has also been widely used in the assessment of r-OSA in the literature.

According to the classification proposed by Hirshkowitz and Sharafkhaneh [23], the pressure calculated by formula in our study obtained a mean value of residual $\mathrm{AHI}$ in supine REM of 8.4 events/h, with a mean of 44.1 min of supine REM sleep. This pressure could be classified as good quality pressure (acceptable pressure).

The evaluated formula is not fully able to establish an optimal pressure for the treatment $[8,9]$. Many patients do not achieve optimal pressure settings, and post-hoc analyses of randomized trials have shown that only 50 to $60 \%$ of patients are able to achieve optimal or good pressure (acceptable pressure), while 30 to $40 \%$ are classified as having adequate pressure or inadequate (unacceptable pressure) during manual titration [13-15].
Our data showed that $27 \%$ of the patients were considered to have adequate and inadequate pressure quality, while $73 \%$ achieved the optimal and good grading level. A titration for CPAP pressure assessment should be repeated if optimal or good quality (acceptable quality) is not reached. The results of manual titration polysomnography presented by $\mathrm{Su}$ et al. were divided into $52.7 \%$ with optimal pressure, $0.5 \%$ with good pressure, $38.9 \%$ with adequate pressure, and $7.9 \%$ with inadequate pressure, making a total of $53.2 \%$ of acceptable exams (optimal and good pressure) and $46.8 \%$ of unacceptable exams, which would need to be repeated [15].

When the data collection and research follow-up protocol ended, in cases of general residual AHI above 5 events/h, there was an empirical adjustment of +1 to $+2 \mathrm{cmH}_{2} \mathrm{O}$ in the calculated pressure, until the performance of manual titration polysomnography according to the routine of the outpatient care follow-up.

There are questions about the impact of a general residual AHI just above 5 events/h and possible cardiovascular risk. The threshold at which OSA becomes a disease (reducing longevity or quality of life) is unknown, just as it is not known with certainty how problematic residual events are [24]. It should be noted that it is unclear which cut-off point is critical for improving outcomes after CPAP treatment.

Epidemiological studies indicate that cardiovascular disease is more likely to occur in patients with an $\mathrm{AHI} \geq 10 / \mathrm{h}$; however, an AHI between 5 and 10 has also been associated with the development of arterial hypertension. Other studies have shown that the $\mathrm{AHI}$ is an independent cardiovascular and traffic accident risk factor, but the long-term implications of residual apneas and hypopneas after treatment with CPAP have not been established [12].

The method of determining the CPAP pressure plays an important role in improving the patient's clinical condition. However, it is uncertain whether or not different CPAP titration methods would yield different results if other therapeutic targets, such as cardiovascular risk factors, were taken into account. The effective pressure of CPAP is one that, at the same time, prevents the adverse consequences of OSA and causes minimal discomfort and complications.

A single-night manual CPAP titration is still considered the gold standard for determining effective pressure in the treatment of OSA. However, the test-retest reliability of this measure is questionable [25]. Although it was traditionally considered that night-to-night variability in CPAP requirement was quite low [26], some studies have shown that CPAP requirement may have more variability than is often recognized [25].

In a study published in 2021, Farré, Gozal, and Montserrat reviewed the literature and suggested a much more 
Table 2 Sleep characteristics and polysomnography $(n=80)$

\begin{tabular}{|c|c|c|c|}
\hline \multirow[t]{2}{*}{ Variable } & \multicolumn{2}{|c|}{$N(\%)$ or mean $(\mathrm{sd})$} & \multirow[t]{2}{*}{ p-value ${ }^{\#}$} \\
\hline & Baseline & Intervention & \\
\hline \multicolumn{4}{|l|}{ Sleep position* } \\
\hline Lateral & $45(56 \%)$ & $57(71 \%)$ & 0.0047 \\
\hline Supine & $35(44 \%)$ & $23(29 \%)$ & \\
\hline \multicolumn{4}{|l|}{ Snoring ${ }^{*}$} \\
\hline No & $1(1 \%)$ & $77(96 \%)$ & $<0.0001$ \\
\hline Yes & $79(99 \%)$ & $3(4 \%)$ & \\
\hline Sleep Efficiency (\%) & $77.8 \pm 12.7$ & $86.9 \pm 9.4$ & $<0.0001$ \\
\hline Sleep Onset (min) & $14.5 \pm 15.1$ & $9.9 \pm 9.0$ & 0.0272 \\
\hline REM latency (min) & $147.5 \pm 96.2$ & $177.3 \pm 96.2$ & 0.0482 \\
\hline N1\% & $28.4 \pm 17.5$ & $16.0 \pm 10.7$ & $<0.0001$ \\
\hline $\mathrm{N} 2 \%$ & $42.3 \pm 14.2$ & $54.2 \pm 12.3$ & $<0.0001$ \\
\hline N3\% & $15.6 \pm 11.6$ & $14.7 \pm 9.2$ & 0.6167 \\
\hline REM \% & $13.6 \pm 7.4$ & $15.0 \pm 6.9$ & 0.2349 \\
\hline AHI (events/h) & $49.5 \pm 27.3$ & $6.1 \pm 5.2$ & $<0.0001$ \\
\hline Supine position AHI & $55.1 \pm 32.2$ & $7.2 \pm 7.3$ & $<0.0001$ \\
\hline Left position AHI & $41.7 \pm 37.0$ & $1.6 \pm 2.2$ & $<0.0001$ \\
\hline Right position AHI & $34.3 \pm 33.9$ & $2.5 \pm 3.6$ & $<0.0001$ \\
\hline REM sleep AHI & $52.3 \pm 35.4$ & $9.5 \pm 14.2$ & $<0.0001$ \\
\hline $\begin{array}{l}\text { Respiratory arousal } \\
\text { (events/h) }\end{array}$ & $40.9 \pm 30.2$ & $1.9 \pm 2.2$ & $<0.0001$ \\
\hline Arousal index (events/h) & $57.6 \pm 27.1$ & $14.5 \pm 11.0$ & $<0.0001$ \\
\hline Leg arousal (events/h) & $1.4 \pm 2.4$ & $1.5 \pm 2.5$ & 0.8378 \\
\hline PLMs (events/h) & $12.9 \pm 25.7$ & $7.3 \pm 13.6$ & 0.1484 \\
\hline NREM Saturation (\%) & $92.4 \pm 3.2$ & $95.1 \pm 2.0$ & $<0.0001$ \\
\hline REM Saturation (\%) & $90.8 \pm 4.8$ & $95.0 \pm 2.3$ & $<0.0001$ \\
\hline Mean Saturation (\%) & $92.1 \pm 3.4$ & $95.0 \pm 2.4$ & $<0.0001$ \\
\hline T90 (\%) & $15.6 \pm 20.7$ & $2.3 \pm 8.2$ & $<0.0001$ \\
\hline
\end{tabular}

\# $p$-value calculated by McNemar's test, paired Student's $t$ or Wilcoxon; *sleep characteristics at home at baseline and after 30 days of treatment. REM, rapid eyes movement; $A H I$, apnea and hypopnea index; PLM, periodic limb movement; NREM, non rapid eyes movement; $T 90$, sleep time with saturation below $90 \%$

simplistic approach to initial treatment with CPAP at a pressure of $9 \mathrm{cmH}_{2} \mathrm{O}$, which would subsequently be followed by any necessary adjustments in only a minority of patients who did not obtain the necessary benefit from such a standardized intervention [27].

Outpatient follow-up should be performed with monitoring of daytime sleepiness, quality of life, and blood pressure reduction in hypertensive patients with OSA. While several authors use the reduction of the residual AHI to below 10/h as an effective treatment parameter, the reduction to values below $5 / \mathrm{h}$ may be necessary to reverse daytime sleepiness [28]. Furthermore, there is a significant effect even in subtherapeutic pressure with CPAP on Epworth scale measures [29].

Our baseline ESS data decreased by 5.2 and 5.9 points at each reassessment. Lloberes et al. showed a reduction of 7.4
Table 3 Association between clinical variables and acceptable CPAP pressure

\begin{tabular}{lllll}
\hline & Gross PR & \multicolumn{3}{l}{ Adjusted PR } \\
\hline $\begin{array}{l}\text { Variable } \\
\text { Age (years) }\end{array}$ & PR $(C I 95 \%)$ & $p$-value & PR $(C I 95 \%)$ & $p$-value \\
$<60$ & 1 & - & - & - \\
$\geq 60$ & $1.10(0.84 ; 1.45)$ & 0.4659 & - & - \\
Sex & & & & - \\
Female & 1 & - & - & - \\
Male & $1.13(0.86 ; 1.48)$ & 0.3882 & - & - \\
Weight $(\mathrm{kg})$ & & & & 0.0008 \\
$<105$ & 1 & - & 1 & \\
$\geq 105$ & $1.44(1.17 ; 1.78)$ & 0.0006 & $1.43(1.16 ; 1.77)$ & 0. \\
\hline
\end{tabular}

Cervical circumference

$\begin{array}{lllll}\leq 46(\mathrm{~cm}) & 1 & - & - & - \\ >46(\mathrm{~cm}) & 1.34(1.07 ; 1.68) & 0.0101 & - & - \\ \begin{array}{l}\text { Type of mask } \\ \text { Oronasal }\end{array} & 1 & & & - \\ \text { Nasal } & 1.69(1.00 ; 2.84) & 0.0487 & 1.81(1.17 ; 2.79) & 0.0076 \\ \text { Asthma } & & & & \\ \text { No } & 1 & - & - & - \\ \text { Yes } & 1.15(0.84 ; 1.58) & 0.3809 & - & - \\ \text { Diabetes } & & & & - \\ \text { No } & 1 & - & 1 & 0.0120 \\ \text { Yes } & 1.38(1.07 ; 1.77) & 0.0132 & 1.35(1.07 ; 1.70) & \end{array}$

Systematic hypertension

$\begin{array}{llll}\text { No } & 1 & - & - \\ \text { Yes } & 1.26(0.92 ; 1.74) & 0.1504 & -\end{array}$

Benzodiazepine

No $\quad 1.29(0.78 ; 2.11) \quad 0.3221 \quad-$

Yes 1

m-Mallampati

Grade $1-2 \quad 1$

Grade 3 -4 $1.06(0.78 ; 1.45) \quad 0.7175 \quad$ -

ESS 30 day-assessment

$\begin{array}{lllll}\leq 10 & 1.60(1.01 ; 2.52) & 0.0434 & 1.58(1.08 ; 2.30) & 0.0178 \\ >10 & 1 & - & 1 & 1\end{array}$

ESS 120 day-assessment-

$\leq 10 \quad 1.20(0.80 ; 1.80) \quad 0.3777 \quad-$

$>10 \quad 1$

*Distribution of study variables according to the gross and adjusted prevalence ratios calculated by the Poisson regression model with robust variance and their respective $95 \%$ confidence intervals. $P R$, prevalence ratio; CI 95\%, 95\% confidence interval; ESS 30 dayassessment, Epworth scale value in the first outpatient assessment after 30 days of treatment; ESS 120 day-assessment, Epworth scale value in the second outpatient assessment after 120 days of treatment

points in the ESS in patients treated with manually titrated pressure of $7.5 \pm 2.2 \mathrm{cmH}_{2} \mathrm{O}$ [20], close to the mean pressure obtained by the formula in our study $\left(7.8 \pm 2.1 \mathrm{cmH}_{2} \mathrm{O}\right)$. 
Table 4 Association of AHI $<5$ events/hour and anthropometric, clinical, mask type and adherence to CPAP variables

\begin{tabular}{|c|c|c|c|c|}
\hline & Gross PR* & & Adjusted $\mathrm{PR}^{*}$ & \\
\hline Variable & $\mathrm{PR}(C I 95 \%)$ & $p$-value & $\mathrm{PR}(C I 95 \%)$ & $p$-value \\
\hline \multicolumn{5}{|l|}{ Age (years) } \\
\hline$<60$ & 1 & - & & \\
\hline$\geq 60$ & $1.10(0.71 ; 1.71)$ & 0.6540 & & \\
\hline \multicolumn{5}{|l|}{ Sex } \\
\hline Female & 1 & - & & \\
\hline Male & $1.16(0.75 ; 1.80)$ & 0.5030 & & \\
\hline \multicolumn{5}{|l|}{ Weight (kg) } \\
\hline$<105$ & 1 & - & & \\
\hline$\geq 105$ & $1.07(0.65 ; 1.76)$ & 0.7891 & & \\
\hline \multicolumn{5}{|c|}{ Cervical circumference } \\
\hline$\leq 46(\mathrm{~cm})$ & 1 & - & & \\
\hline$>46(\mathrm{~cm})$ & $1.09(0.62 ; 1.91)$ & 0.7546 & & \\
\hline \multicolumn{5}{|c|}{ Type of mask } \\
\hline Oronasal & 1 & - & & \\
\hline Nasal & $1.89(0.88 ; 4.08)$ & 0.1050 & & \\
\hline \multicolumn{5}{|l|}{ Asthma } \\
\hline No & 1 & - & 1 & - \\
\hline Yes & $1.82(1.24 ; 2.67)$ & 0.0021 & $1.79(1.18 ; 2.70)$ & 0.0057 \\
\hline \multicolumn{5}{|l|}{ Diabetes } \\
\hline Não & 1 & - & & \\
\hline Sim & $1.17(0.75 ; 1.81)$ & 0.4851 & & \\
\hline \multicolumn{5}{|c|}{ Systemic hypertension } \\
\hline No & 1 & - & & \\
\hline Yes & $0.95(0.60 ; 1.48)$ & 0.8148 & & \\
\hline \multicolumn{5}{|c|}{ Benzodiazepine } \\
\hline No & $0.83(0.49 ; 1.42)$ & 0.5020 & & \\
\hline Yes & 1 & & & \\
\hline \multicolumn{5}{|c|}{ m-Mallampati } \\
\hline Grade 1,2 & 1 & - & & \\
\hline Grade 3,4 & $0.84(0.53 ; 1.32)$ & 0.8381 & & \\
\hline \multicolumn{5}{|c|}{ ESS 30 day-assessment } \\
\hline$\leq 10$ & $1.15(0.67 ; 1.98)$ & 0.6179 & & \\
\hline$>10$ & 1 & - & & \\
\hline \multicolumn{5}{|c|}{ ESS 120 day-assessment } \\
\hline$\leq 10$ & $0.75(0.47 ; 1.19)$ & 0.2208 & & \\
\hline$>10$ & 1 & - & & \\
\hline \multicolumn{5}{|c|}{ Adherence CPAP 30 day-assessment } \\
\hline$<7 \mathrm{~h}$ & 1 & - & & \\
\hline$>7 \mathrm{~h}$ & $1.29(0.78 ; 2.12)$ & 0.3179 & & \\
\hline \multicolumn{5}{|c|}{ Adherence CPAP 120 day-assessment } \\
\hline$<7 \mathrm{~h}$ & 1 & - & & \\
\hline$>7 \mathrm{~h}$ & $1.00(0.52 ; 1.94)$ & 1.0000 & & \\
\hline
\end{tabular}

*Distribution of study variables according to the gross and adjusted prevalence ratios calculated by the Poisson regression model with robust variance and their respective $95 \%$ confidence intervals. $P R$, prevalence ratio; CI 95\%, 95\% confidence interval; ESS 30 dayassessment, Epworth scale value in the first outpatient assessment after 30 days of treatment; ESS 120 day-assessment, Epworth scale value in the second outpatient assessment after 120 days of treatment
Table 5 Comparison of variables between patients with residual AHI less than or equal to 10 events/h and residual AHI greater than 10 events/h

\begin{tabular}{|c|c|c|c|}
\hline \multicolumn{4}{|l|}{ Residual AHI } \\
\hline Variables & $\begin{array}{l}\mathrm{r}-\mathrm{AHI} \leq 10 \\
n=65\end{array}$ & $\begin{array}{l}\mathrm{r}-\mathrm{AHI}>10 \\
n=15\end{array}$ & $p$-value \\
\hline Sex, $n(\%)$ & & & 0.18 \\
\hline Female & $31(76)$ & $10(24)$ & \\
\hline Male & $34(87)$ & $5(13)$ & \\
\hline Age, years & $58.2(11.9)$ & $60.4(8.4)$ & 0.50 \\
\hline Weight, kg & $93.7(23.1)$ & $82.2(19.0)$ & 0.07 \\
\hline Height, $\mathrm{cm}$ & $165.2(10.5)$ & $158.4(9.8)$ & 0.02 \\
\hline BMI, $\mathrm{kg} / \mathrm{m}^{2}$ & $34.4(7.6)$ & $32,8(7.3)$ & 0.45 \\
\hline $\mathrm{CC}, \mathrm{cm}$ & $42.5(3.9)$ & $40.1(4.8)$ & 0.04 \\
\hline Baseline AHI, n/h & $48.0(28.3)$ & $55.5(22.5)$ & 0.34 \\
\hline Pressure, $\mathrm{cmH}_{2} \mathrm{O}$ & $7.9(2.2)$ & $7.8(2.0)$ & 0.95 \\
\hline Nocturnal $\mathrm{SpO}_{2}$ with CPAP, $\%$ & $96.9(1.7)$ & $97.7(1.6)$ & 0.05 \\
\hline Baseline ESS, points & $11.5(5.6)$ & $14.1(5.5)$ & 0.10 \\
\hline ESS 30 day-assessment, points & $7.1(5.7)$ & $6.1(6.4)$ & 0.55 \\
\hline ESS 120 day-assessment, points & $6.2(5.0)$ & $5.7(4.6)$ & 0.71 \\
\hline
\end{tabular}

Data expressed as mean (standard deviation), unless otherwise indicated. BMI, body mass index; $C C$, cervical circumference; $A H I$, apnea and hypopnea index; $S p O 2$, oxygen saturation; $C P A P$, continuous positive airway pressure; ESS, Epworth sleepiness scale. The $p$-value refers to Student's $t$ test or Mann-Whitney

Patients using pressure considered optimal or good (acceptable pressure) were 3.61 times more likely to obtain ESS $<10$ in outpatient follow-up. This association is suggested by the authors to be used as a potential predictor of acceptable pressure estimated by the formula at the time of outpatient assessment during the 30-day follow-up. AASM guidelines suggest that titration polysomnography is not indicated for patients apparently satisfied with treatment initiated with pressure defined by other methods [3].

While the general residual AHI is determined by the total number of residual apneas and hypopneas in relation to the total sleep time, composed of the 4 sleep stages (N1, N2, N3, and REM) in the different decubitus positions throughout sleep (supine, lateral, and prone), the residual AHI related to acceptable/unacceptable pressure is not determined by the measurement of the general residual AHI, or only in the REM stage, but by the specific measure of the residual AHI present in the REM-supine association. It is the overcoming of the critical level of closure of the upper airways against the simultaneous sum of the two strongest external factors: the supine REM. These findings add further evidence in favor of the major contribution of the 2005 classification system and which was later adopted in the 2008 manual titration guidelines [3,23]. A significant proportion of REM sleep may require higher blood pressure [15]. Furthermore, the positional component between the supine and lateral 
Table 6 Odds ratio for ESS and r-AHI

\begin{tabular}{|c|c|c|c|c|c|}
\hline & & Univariate & & Multivariate $^{*}$ & \\
\hline ESS & $\begin{array}{l}\text { r-AHI } \\
\text { (events/h) }\end{array}$ & $O R(C I 95 \%)$ & $p$-value & $\begin{array}{l}\text { OR } \\
(C I 95 \%)\end{array}$ & $p$-value \\
\hline \multirow{3}{*}{$\begin{array}{c}\text { No daytime } \\
\text { sleepiness } \\
(\mathrm{ESS}<10)\end{array}$} & $\mathrm{r}-\mathrm{AHI}<5$ & $1.15(0.46 ; 2.86)$ & 0.7579 & $\begin{array}{l}0.83 \\
(0.30 ; 2.33)\end{array}$ & 0.7193 \\
\hline & $\mathrm{r}-\mathrm{AHI}<15$ & $1.49(0.17 ; 14.29)$ & 0.7214 & $\begin{array}{l}1.22 \\
(0.18 ; 8.33)\end{array}$ & 0.8384 \\
\hline & $\begin{array}{l}\text { Acceptable pres- } \\
\text { sure AHI }\end{array}$ & $2.81(1.07 ; 7.44)$ & 0.0368 & $\begin{array}{l}3.61 \\
(1.30 ; 10.07)\end{array}$ & 0.0138 \\
\hline
\end{tabular}

*Adjusted by CPAP adherence, use of benzodiazepines, sleep efficiency in intervention polysomnography; ESS, Epworth sleepiness scale; $r$ - $A H I$, residual apnea and hypopnea index; OR, odds ratio; $C I$ 95\%, $95 \%$ confidence interval

Table 7 Longitudinal changes in ESS

\begin{tabular}{lllllll}
\hline & Moment & & & \multicolumn{2}{c}{$\begin{array}{l}p \text {-value } \\
\text { Comparison between time-points }\end{array}$} \\
\hline Variable & Baseline & Change to 30 day- assessment & Change to 120 day- assessment & Baseline $\times 30 \mathrm{~d}$ & Baseline $\times 120 \mathrm{~d}$ & $30 \times 120 \mathrm{days}$ \\
ESS & $12.0 \pm 0.6$ & $-5.1 \pm 0.6$ & $-5.9 \pm 0.5$ & $<0.0001$ & $<0.0001$ & 0.2619 \\
\hline
\end{tabular}

${ }^{\#} p$-value was calculated by linear models of mixed effects; Ess, Epworth sleepiness scale

position can be used as an argument for using the supine AHI or the supine REM AHI of baseline polysomnography in the predictive formulas instead of the general AHI [30].

The main limitations of our study are the absence of a control group with autoCPAP or manual titration and the use of an old and outdated formula, associated with doubts in the literature about the cut-off point for residual AHI during treatment with positive pressure.

In conclusion, the data from this study show that a formula provides an effective initial pressure in the majority of patients (73\%) on treatment without prior APAP or manual titration. This simplified approach is a viable alternative, with reduced waiting lists and time from diagnosis to initiation of therapy. Our results are of significant value in evaluating the effectiveness of treatment with a programmed CPAP from the pressure estimated by the formula. At this moment of the COVID-19 pandemic and restriction to polysomnographic studies in the sleep laboratory, these data gain additional value.

Acknowledgements The authors would like to thank the technical staff of the Sleep Laboratory of the Respiratory Division Luciana Straccia, Iris Sena, and Alessandro Vilela for their assistance in performing the polysomnography exams. We would also like to thank Geruza A. Silva, $\mathrm{MD}$, for the help and advices during protocol planning.

Author contribution All authors contributed to the study conception and design. Material preparation, data collection, and analysis were performed by Danilo Martins de Sá. The first draft of the manuscript was written by Danilo Martins de Sá and all authors commented on previous versions of the manuscript. All authors read and approved the final manuscript.
Funding This study was financed in part by the Coordenação de Aperfeiçoamento de Pessoal de Nível Superior-Brasil (CAPES)—Finance Code 001.

Data Availability All authors certify that all data and materials support their published statements and comply with field standards.

Code availability Not applicable for that section.

\section{Declarations}

Ethics approval This study was performed in line with the principles of the Declaration of Helsinki. Approval was granted by the Ethics Committee of Medical School of Ribeirão Preto, University of São Paulo, Brazil, Date May 07, 2018, No. 2.638.894.

Consent to participate Informed consent was obtained from all individual participants included in the study.

Conflict of interest The authors declare that they have no conflict of interests.

\section{References}

1. Sateia MJ (2014) International Classification of Sleep Disorders. 3rd ed. Copyright $\odot 2014$ American Academy of Sleep Medicine, 2510 North Frontage Road, Darien, IL 60561, U.S.A. Copies of the manual are available from the American Academy of Sleep Medicine in the U.S.A. ISBN:0991543416 (print) ISBN: 0991543408.

2. Epstein LJ, Kristo D, Strollo PJ Jr, Friedman N et al (2009) Adult obstructive sleep apnea task force of the american academy of sleep medicine: clinical guideline for the evaluation, management 
and long-term care of obstructive sleep apnea in adults. J Clin Sleep Med 15;5(3):263-276

3. Kushida CA, Chediak A, Berry RB et al (2008) Clinical guidelines for the manual titration of positive airway pressure in patients with obstructive sleep apnea. positive airway pressure titration task force of the american academy of sleep medicine. J Clin Sleep Med 4(2):157-171

4. Morgenthaler T, Aurora RN, Brown T et al (2008) Practice parameters for the use of autotitrating continuous positive airway pressure devices for titrating pressures and treating adult patients with obstructive sleep apnea syndrome: an update for 2007. An American Academy of Sleep Medicine Report. Sleep 31:141-147

5. Hukins CA (2005) Arbitrary-pressure continuous positive airway pressure for obstructive sleep apnea syndrome. Am J Respir Crit Care Med 171:500-505. https://doi.org/10.1164/rccm. 200401-019OC

6. Skomro RP, Cotton DJ, Gjevre JA, Grover VK, McNab BD, Reid JK, Ward HA (2007) An empirical continuous positive airway pressure trial for suspected obstructive sleep apnea. Can Respir J 14(3):159-163

7. Camacho M, Riaz M, Tahoori A, Certal V, Kushida CA (2015) Mathematical equations to predict positive airway pressures for obstructive sleep apnea: a systematic review. Sleep Disorders. Article ID 293868. https://doi.org/10.1155/2015/293868

8. Miljeteig H, Hoffstein V (1993) Determinants of continuous positive airway pressure level for treatment of obstructive sleep apnea. Am Rev Respir Dis 147:1526-1530

9. Hoffstein V, Mateika S (1994) Predicting nasal continuous positive airway pressure. Am J Respir Crit Care Med 150(2):486-488

10. Schiza SE, Bouloukaki I, Mermigkis C et al (2011) Utility of formulas predicting the optimal nasal continuous positive airway pressure in a Greek population. Sleep Breath 15:417-423. https:// doi.org/10.1007/s11325-010-0352-5

11. Gokcebay N et al (1996) Accuracy of CPAP predicted from anthropometric and polysomnographic indices. Sleep 19(7):600-601

12. Masa JF, Jimenez A, Duran J, Capote F, Monasterio C, Mayos $M$ et al (2004) Alternative methods of titrating continuous positive airway pressure: a large multicenter study. Am J Respir Crit Care Med 170:1218-1224. https://doi.org/10.1164/rccm. 200312-1787OC

13. Freedman N, Kuzniar TJ (2017) Mode selection for positive airway pressure titration in adults with obstructive sleep apnea. UpToDate ${ }^{\circledR}$. Disponível em: https://www.uptodate.com/conte nts/mode-selection-for-titration-of-positive-airway-pressure-inadults-with-obstructive-sleep-apnea. Literature review current through: May 2017. Acesso em: 01 jun 2017.

14. LETTIERI CJ, et al (2008) Eszopiclone improves overnight polysomnography and continuous positive airway pressure titration: a prospective, randomized, placebo-controlled trial. Sleep 31(9):1310-1316

15. Su CS et al (2012) Functional outcomes in patients with REM related obstructive sleep apnea treated with positive airway pressure therapy. J Clin Sleep Med 8(3):243-247

16. American Academy of Sleep Medicine (AASM) (1999) Sleeprelated breathing disorders in adults: recommendations for syndrome definition and measurement techniques in clinical research.
The Report of an American Academy of Sleep Medicine Task Sleep 22(2):667-689

17. Zou G (2004) A modified poisson regression approach to prospective studies with binary data. Am J Epidemiol 159:702-706

18. Schwab RJ, Badr SM, Epstein LJ et al (2013) The optimal monitoring strategies and outcome measures in adults ATS subcommittee on CPAP adherence tracking systems. Am J Respir Crit Care Med 188(5):613-620. https://doi.org/10.1164/rccm. 201307-1282ST

19. Berry RB, Kushida CA, Kryger MH, Soto-Calderon H, Staley B, Kuna ST (2012) Respiratory event detection by a positive airway pressure device. Sleep 35(3):361-367. https://doi.org/10.5665/ sleep. 1696

20. Lloberes P, Ballester E, Montserrat JM, Botifoll E, Ramirez A, Reolid A, Gistau C, Rodriguez-Roisin R (1996) Comparison of manual and automatic cpap titration in patients with sleep apnea/ hypopnea syndrome. Am J Resp Crit Care Med 154:1755-1758

21. Issa FG, Sullivan CE (1986) The immediate effects of nasal continuous positive airway pressure treatment on sleep pattern in patients with obstructive sleep apnea syndrome. Electroencephalogr Clin Neurophysiol 63(1):10-17

22. Desai H, Patel A, Patel P, Grant BJ, Mador MJ (2009) Accuracy of autotitrating CPAP to estimate the residual apnea-hypopnea index in patients with obstructive sleep apnea on treatment with autotitrating CPAP. Sleep Breath 13:383-390

23. Hirshkowitz M, Sharafkhaneh A (2005) Positive airway pressure therapy of OSA sleep and respiration. Editor Seminars in Respiratory and Critical Care Medicine 26(1):68-79

24. Beninati W, Sanders MH (2001) Optimal continuous positive airway pressure for the treatment of obstructive sleep apnea/hypopnea. Sleep Med Rev 5(1):7-23

25. Netzer NC et al (2011) The need for pressure changes in CPAP therapy $2-3$ months after initial treatment: a prospective trial in 905 patients with sleep-disordered breathing. Sleep and Breathing 15(1):107-112

26. Choi $\mathrm{S}$ et al (2001) Is (re)titration of nasal continuous positive airway pressure for obstructive sleep apnoea necessary? Sleep Med 2(5):431-435

27. Farré R, Gozal D, Montserrat JM (2021) Alternative procedure to individual nasal pressure titration for sleep apnea. J Clin Med 7:1453-1459

28. Montserrat JM, Ballester E, Olivi H (1995) Time course of stepwise CPAP titration: behavior of respiratory and neurological variables. Am J Respir Crit Care Med 152:1854-1859

29. Jenkinson C et al (1999) Comparison of therapeutic and subtherapeutic nasal continuous positive airway pressure for obstructive sleep apnoea: a randomised prospective parallel trial. Lancet 353(9170):2100-2105

30. Lai CC et al (2015) Clinical predictors of effective continuous positive airway pressure in patients with obstructive sleep apnea/ hypopnea syndrome. Laryngoscope 125(8):1983-1987

Publisher's Note Springer Nature remains neutral with regard to jurisdictional claims in published maps and institutional affiliations. 